\title{
Editorial
}

\section{Food Chemistry: Food Quality and New Analytical Approaches}

\author{
Nivia M. M. Coelho, ${ }^{1}$ César R. T. Tarley, ${ }^{2}$ Claudia Ruiz-Capillas, ${ }^{3}$ \\ Luciana M. Coelho, ${ }^{4}$ and Miguel de la Guardia ${ }^{5}$ \\ ${ }^{1}$ Federal University of Uberlândia, 38400902 Uberlândia, MG, Brazil \\ ${ }^{2}$ State University of Londrina, 86057970 Londrina, PR, Brazil \\ ${ }^{3}$ Instituto de Ciencia y Tecnología de Alimentos y Nutrición (ICTAN), 28040 Madrid, Spain \\ ${ }^{4}$ Federal University of Goiás, 75704020 Catalão, GO, Brazil \\ ${ }^{5}$ Department of Analytical Chemistry, University of Valencia, 46100 Valencia, Spain
}

Correspondence should be addressed to Nivia M. M. Coelho; nmmcoelho@ufu.br

Received 18 March 2016; Accepted 22 March 2016

Copyright ( 92016 Nivia M. M. Coelho et al. This is an open access article distributed under the Creative Commons Attribution License, which permits unrestricted use, distribution, and reproduction in any medium, provided the original work is properly cited.

Foods, their production systems, and quality control are challenging subjects of the today research in an increasing population world and, because of that, the scientific community has made big efforts to improve the analytical tools to effectively control the quality and safety of human foods. That, which is a main topic in the developed countries, merits too special attention in developing areas in order to extend the safety to all the steps of the production of foods and all consumed products all around the world. So, this special issue if devoted to food chemistry: food quality and new analytical approaches.

The continuous efforts of food industries to improve their strategies of production, transformation, and distribution of foods have been focused on the development of new technologies for food processing, which aimed at the enhancement of food properties or to create novel products suitable for covering the needs of consumers and for guaranteeing safety, health, and well accepted standards.

In the aforementioned frame, food industry needs to improve chemical and biological tools suitable for guaranteeing a safe production. To do that, microbiological and chemical methods of analysis have an increasing value as control systems. However, methods available nowadays for a fast and continuous monitoring of the main food components, additives, and contaminants are still scarce and not in all cases are we suitable for solving the problems created by traditional contaminants as heavy metals, pesticides, pharmaceutical residues, and emerging contaminants. In fact there are serious worries in all countries about the presence of pesticides in cereals, vegetables, and milk and dairy products, the contamination of fishes by heavy metals, like mercury, lead, cadmium, or arsenic, between others, or the presence of pharmaceutical residues in farm meat products. So, new approaches are required for food sampling, sample preparation, and analysis of chemical components, their metabolites, and mineral species in foods.

There are inherent difficulties associated with the different types of food samples involved in human nutrition and variations in their composition as a function of the production and industrialization conditions. Additionally, there is a growing interest for the improvement of food properties, food authentication, which is especially concerned with the so-called organic foods and protected designation of origin foods, and/or to produce novel products, as well as for the systematic analysis of contaminants or additives in produced foods to be exported to foreign countries and also to be consumed in the same country and, because of that, not only food producers but also consumers and policy makers are forced to develop accurate, precise, sensitive, and selective tools for a fast control of too many parameters in foods.

This special issue aims to present the most recent advances in the field of food chemistry, which have been achieved in recent years as a consequence of recent progress in food quality requirements and new analytical approaches available in the field.

The topics included in this issue have been properly selected and concern new research challenges in food quality and safety control, general food and beverages analysis, food 
forensics, bioactive food constituents and functions determination, chemical changes induced by food processing and storage, recent advances in sample preparation techniques in the food field, and chemical speciation in foods.

\section{Acknowledgments}

We are would like to deeply acknowledge the authors, who submitted their excellent contributions, and the editorial staff, who helped us to improve the submitted manuscripts. Thus, based on the aforementioned efforts, we are convinced that the readers will find in this special issue many topics and works of interest for their daily practice and information and we hope that this work could become a reference text for the future research efforts in this field. So, we offer many thanks for reading the enclosed papers and our best wishes for your future research.

Nivia M. M. Coelho

César R. T. Tarley

Claudia Ruiz-Capillas

Luciana M. Coelho

Miguel de la Guardia 

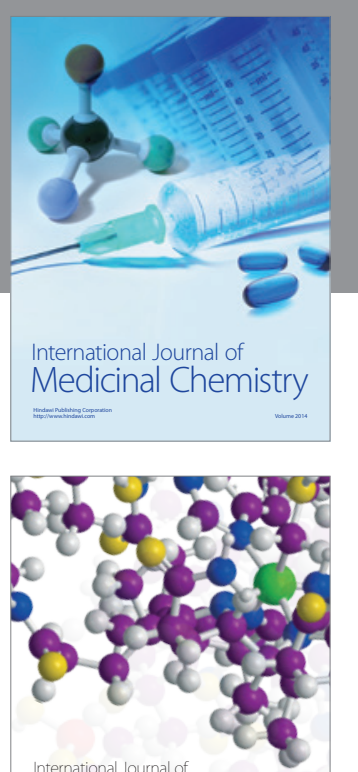

Carbohydrate Chemistry

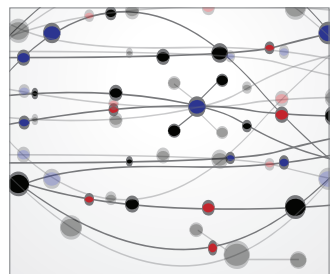

The Scientific World Journal
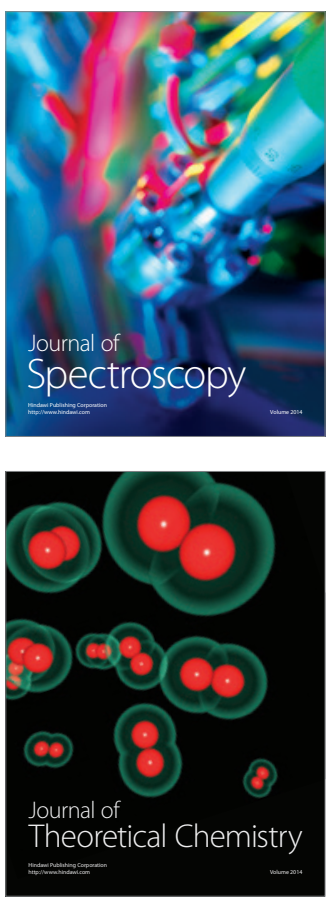
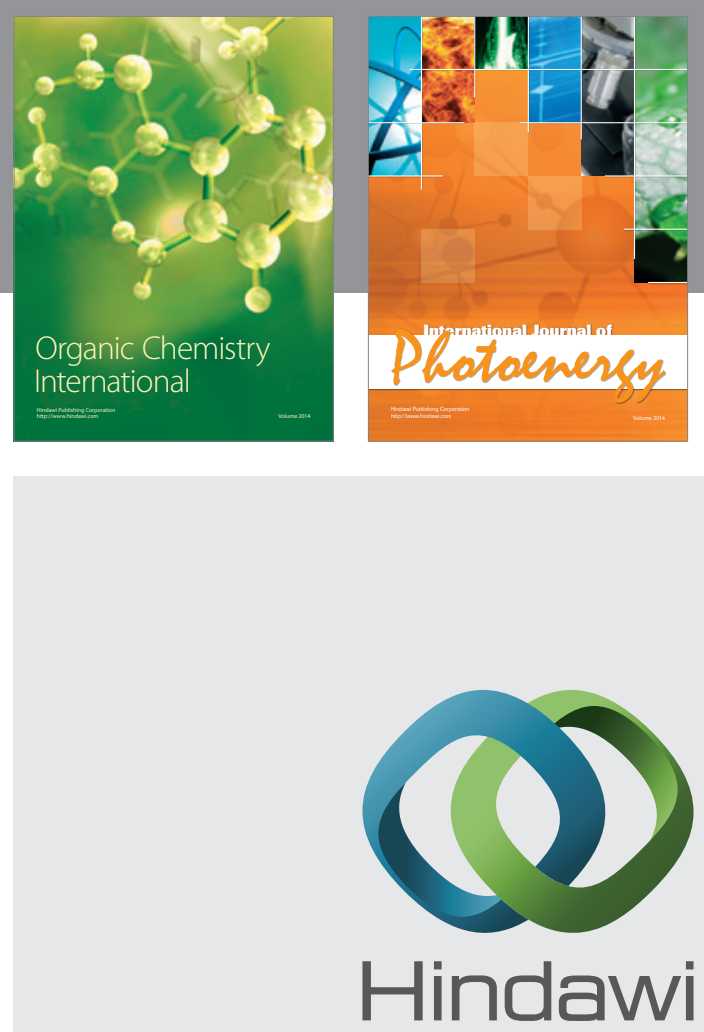

Submit your manuscripts at

http://www.hindawi.com

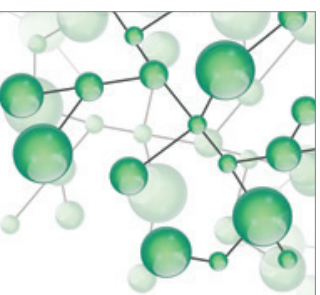

International Journal of

Inorganic Chemistry

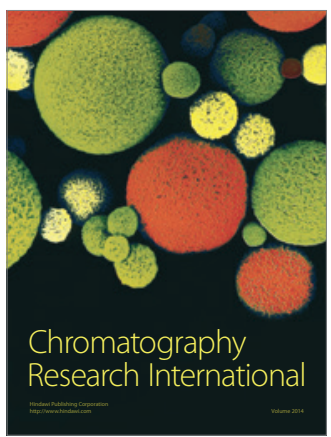

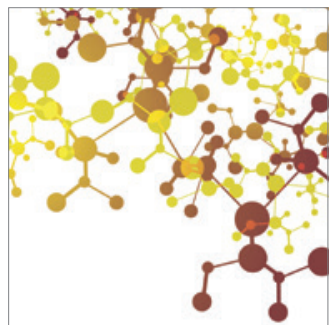

Applied Chemistry
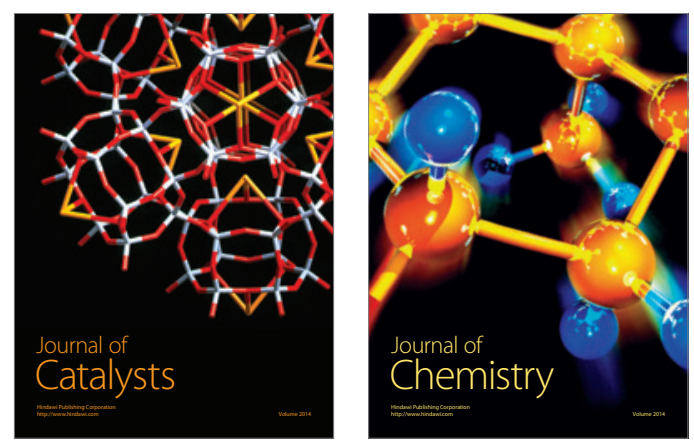
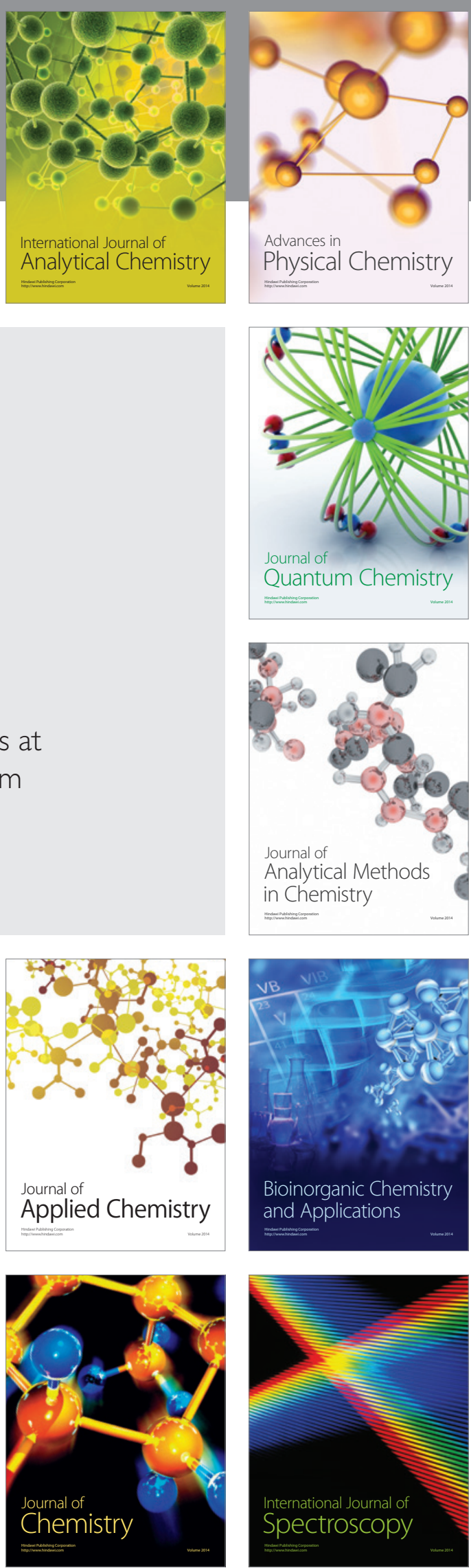